\title{
Solving an Asian option PDE via the Laplace transform
}

\author{
Zieneb Ali Elshegmani ${ }^{\mathrm{a}, *}$, Rokiah Rozita Ahmad ${ }^{\mathrm{b}}$ \\ a School of Mathematical Sciences, Faculty of Education, University Misurata Libya, University Kebangsaan, \\ Malaysia \\ b School of Mathematical Sciences, Faculty of Science and Technology, University Kebangsaan, Malaysia \\ *Corresponding author, e-mail: zelsheqmani@yahoo.com
}

Received 7 Jan 2013

Accepted 5 Apr 2013

\begin{abstract}
Solving the Black-Scholes PDE of the arithmetic Asian options is one of the most difficult problems in financial mathematics. A variety of ways ways have been proposed to address the problem. In this study, we use the PDE approach by presenting an efficient method for pricing a continuous arithmetic Asian option. Using the Laplace transform we reduce the three-dimension partial differential equation of the arithmetic Asian option into a two-dimension ordinary differential equation. Its final analytical solution is presented. We conclude that this method is applicable to all types of arithmetic Asian options.
\end{abstract}

KEYWORDS: partial differential equations, arithmetic Asian option

\section{INTRODUCTION}

Asian options are path dependent options whose payoff functions depend on the average stock price over a specific period of time called the life of the option. There are two types of Asian options with regards to the average computation: arithmetic and geometric. Call options give the holder of the option the right to buy an underlying security, but are not obligated to do so, and put options which give the right to sell. For the case of geometric Asian options there is a closed-form solution to the value of these options ${ }^{1}$. However, the most difficult task is the arithmetic type, because the arithmetic average of a set of lognormal random variables is not lognormally distributed. Until now there has been no closed-form solution to the value of these types of option. Some methods have been developed and proposed to address the problem. Geman and Yor $^{2}$ used a Laplace transform in time of the Asian option price. However, this transform is only applicable in some cases. Rogers and $\mathrm{Shi}^{3}$ transformed the problem to the problem of solving a parabolic PDE in two variables from the second order, but there is no analytical solution for this PDE, and its numerical solution is not accurate. They also derive lowerbound formulae for Asian options by computing the expectation based on a zero-mean Gaussian variable. Zhang ${ }^{4}$ presented a theory of continuously-sampled Asian option pricing, and he solved the PDE with a perturbation approach. Vecer's approach ${ }^{5}$ was based on treating the Asian option as an option on a traded account; he provides a one-dimensional PDE for Asian options. Dubois and Lelievre ${ }^{6}$ derived accurate and fast numerical methods to solve the Rogers and Shi PDE. Chen and Lyuu ${ }^{7}$ developed the lower-bound pricing formulae of Rogers and Shi $\mathrm{PDE}^{3}$ to include general maturities instead of for one year. Dewynne and Shaw ${ }^{8}$ provided a simplified means of pricing arithmetic Asian options by the PDE approach, they derive an analytical formula for the Laplace transform in time of the Asian option, and they obtain asymptotic solutions for Black-Scholes PDE for Asian options for the low-volatility limit which is a big problem with using the Laplace transform. Yang et $\mathrm{al}^{9}$ derive quasianalytical expressions for the price and hedge of an arithmetic Asian call option.

Hence, in this study, we provide a simpler direct solution for the arithmetic Asian options using the Laplace transform in time. Using the Laplace transform and a transformation technique on some variables suggested by Rogers and $\mathrm{Shi}^{3}$, we transform the two-dimensional arithmetic Asian option PDE to an ordinary differential equation in one dimension, which is simpler than the equations that have been obtained by previous authors. Our solution requires only one change of some variables, whereas Dewynne and Shaw ${ }^{8}$ and Cruz-Baez and Gonzalez-Rodrigues ${ }^{10}$ applied a series of changes of variables before using the Laplace transform. We use Maple program to get its final analytical solution. 


\section{TECHNIQUES OF TRANSFORMATION}

The Black-Scholes PDE for the arithmetic Asian options is

$$
\frac{\partial V}{\partial t}+\frac{1}{2} \sigma^{2} S^{2} \frac{\partial^{2} V}{\partial S^{2}}+r S \frac{\partial V}{\partial S}+S \frac{\partial V}{\partial A}-r V=0,
$$

with $t \geqslant 0, S>0$ and the boundary conditions

$$
V(T, S, A)=\varphi\left(S, \frac{A}{T}\right) .
$$

$S$ is the stock price, $r$ is the interest rate, and $\sigma$ is the asset volatility. Both $r$ and $\sigma$ are constants. $T$ is the expiration date and $A_{t}=\int_{0}^{t} S(u) \mathrm{d} u$ is a running sum of the stock price.

There are four different types of arithmetic Asian option with regards to the payoff function $\varphi(S, A / T)$ :

(i) Fixed strike call $\varphi(S, A / T)=(k-A / T)^{+}$

(ii) Fixed strike put $\varphi(S, A / T)=((A / T)-k)^{+}$

(iii) Floating strike call $\varphi(S, A / T)=(S-A / T)^{+}$

(iv) Floating strike put $\varphi(S, A / T)=((A / T)-S)^{+}$ where $k$ is the exercise price.

Equation (1) is not easy to solve since the parabolic operator is degenerate $A$. However, it is possible to reduce (1) to a simpler equation using the Laplace transform and transformation techniques on some variables. Using the following steps to transform the variables in (1):

$$
V(t, S, A)=S f(t, \xi), \xi=\frac{k-\frac{A}{T}}{S} .
$$

Eq.(1) is reduced to

$$
\begin{gathered}
\frac{\partial f}{\partial t}-\left(\frac{1}{T}+r \xi\right) \frac{\partial f}{\partial \xi}+\frac{1}{2} \sigma^{2} \xi^{2} \frac{\partial^{2} f}{\partial \xi^{2}}=0 \\
f(T, \xi)=\varphi(\xi) .
\end{gathered}
$$

For the case of fixed strike call option $\varphi(\xi)=$ $\max (-\xi, 0)=\xi^{-}$, fixed strike put option $\varphi(\xi)=$ $\max (\xi, 0)=\xi^{+}$, floating strike call $\varphi(\xi)=$ $\max (\xi, 0)=(1+\xi)^{-}$, and floating strike put $\varphi(\xi)=$ $\max (\xi, 0)=(1+\xi)^{+}$. Also (2) is difficult to solve, because the coefficient $1 / T$ does not multiply by the variable $\xi$.

Rogers and $\mathrm{Shi}^{3}$ obtain the analytical solution of (2) for the case of $\xi \leqslant 0$

$$
f(t, \xi)=\frac{1}{r T}\left(1-\mathrm{e}^{-r(T-t)}\right)-\xi \mathrm{e}^{-r(T-t)} .
$$

Perform the inverse transformation

$$
\begin{aligned}
& V(t, S, A) \\
& \quad=S\left(\frac{1-\mathrm{e}^{-r(T-t)}}{r T}\right)-\mathrm{e}^{-r(T-t)}\left(k-\frac{A}{T}\right) .
\end{aligned}
$$

We shall consider only the case when $\xi>0$, and use (3) to get free boundary condition. Let $\tau=T-t$

$$
\begin{gathered}
\frac{\partial f}{\partial \tau}=\frac{1}{2} \sigma^{2} \xi^{2} \frac{\partial^{2} f}{\partial \xi^{2}}-\left(\frac{1}{T}+r \xi\right) \frac{\partial f}{\partial \xi} \\
f(0, \xi)=\varphi(\xi) \\
f(\tau, 0)=\frac{1}{r T}\left(1-\mathrm{e}^{-r \tau}\right) .
\end{gathered}
$$

In order to avoid the derivation with respect to time and obtain an ODE instead PDE we shall apply the Laplace transform in time $\tau$. We consider the case of fixed strike call option, the initial condition of the fixed strike call Asian option under reducing domain $(\xi>0)$ becomes $f(0, \xi)=0$. Using the Laplace transform and its properties, (4) becomes

$$
\begin{aligned}
p \hat{f} & =\frac{1}{2} \sigma^{2} \xi^{2} \frac{\partial^{2} \hat{f}}{\partial \xi^{2}}-\left(\frac{1}{T}+r \xi\right) \frac{\partial \hat{f}}{\partial \xi}, \\
\hat{f}(p, 0) & =\frac{1}{r T}\left(\frac{r}{p(r+p)}\right) .
\end{aligned}
$$

The solution of (5) can be given as a pair of confluent hypergeometric functions

$$
\begin{gathered}
\hat{f}(p, \xi)=C_{1}(p) h_{1}(p, \xi)+C_{2}(p) h_{2}(p, \xi), \\
h_{1}(p, \xi)=\xi^{\left(\left(r / \sigma^{2}\right)+(1 / 2)-\alpha / 2 \sigma^{2}\right)} \mathrm{e}^{\left((-2) / \sigma^{2} T \xi\right)} \\
\times{ }_{1} F_{1}\left(\frac{3}{2}+\frac{r}{\sigma^{2}}+\frac{\alpha}{2 \sigma^{2}}, \frac{\sigma^{2}+\alpha}{\sigma^{2}}, \frac{2}{\sigma^{2} T \xi}\right), \\
h_{2}(p, \xi)=\xi^{\left(\left(r / \sigma^{2}\right)+(1 / 2)+\alpha / 2 \sigma^{2}\right)} \mathrm{e}^{\left((-2) / \sigma^{2} T \xi\right)} \\
\times{ }_{1} F_{1}\left(\frac{3}{2}+\frac{r}{\sigma^{2}}-\frac{\alpha}{2 \sigma^{2}}, \frac{\sigma^{2}-\alpha}{\sigma^{2}}, \frac{2}{\sigma^{2} T \xi}\right),
\end{gathered}
$$

where $\alpha=\sqrt{\sigma^{4}+4 \sigma^{2}(r+2 p)+4 r^{2}}$. Taking into account the series expansions of the hypergeometric function, this excludes the function $h_{1}(p, \xi)^{8,10}$. And if $\operatorname{Re}(z)<0$, then as $|z| \rightarrow \infty$,

$$
{ }_{1} F_{1}(a, b, z) \sim \frac{\Gamma(b)}{\Gamma(b-a)}(-z)^{-a} .
$$

Using the boundary condition and the above relation we determined $C_{2}(p)$, and then the solution of (5) is given by

$$
\begin{aligned}
& \hat{f}(p, \xi) \\
= & \frac{\xi^{\left(\left(r / \sigma^{2}\right)+(1 / 2)+\alpha / 2 \sigma^{2}\right)} \mathrm{e}^{(-2) / \sigma^{2} T \xi} \Gamma\left(-\frac{1}{2}-\frac{r}{\sigma^{2}}-\frac{\alpha}{2 \sigma^{2}}\right)}{\left(\frac{1}{T p(r+p)}\right) \Gamma\left(\frac{\sigma^{2}-\alpha}{\sigma^{2}}\right)} \\
& \times{ }_{1} F_{1}\left(\frac{3}{2}+\frac{r}{\sigma^{2}}-\frac{\alpha}{2 \sigma^{2}}, \frac{\sigma^{2}-\alpha}{\sigma^{2}}, \frac{2}{\sigma^{2} T \xi}\right) \cdot
\end{aligned}
$$


The final analytical solution for the fixed strike call arithmetic Asian option PDE then, can be obtained using

$$
V(t, S, A)=S f(\tau, \xi), \quad \tau=T-t, \quad \xi=\frac{k-\frac{A}{T}}{S},
$$

where $f(\tau, \xi)$ is given by

$$
\begin{gathered}
f(\tau, \xi)=\frac{1}{r T}\left(1-\mathrm{e}^{-r \tau}\right)-\xi \mathrm{e}^{-r \tau}, \quad \xi \leqslant 0, \\
f(\tau, \xi)=\frac{1}{2 \pi i} \int_{\gamma-i \infty}^{\gamma+i \infty} \hat{f}(p, \xi) \mathrm{e}^{\tau p} \mathrm{~d} p, \quad \xi>0,
\end{gathered}
$$

and $\hat{f}(p, \xi)$ is defined in (6), the integration is done along the vertical line $\operatorname{Re}(p)=\gamma$ in the complex plane such that $\gamma$ is greater than the real part of all singularities of $\hat{f}(p)$ (see Ref. 11). Solution of the fixed strike put option can be obtained using put-call parity.

\section{CONCLUSIONS}

Since the numerical methods for solving arithmetic Asian option PDE is not exact, that is because the low volatility level or short maturity, we tried to solve it analytically by means of partial differential equations. We have shown that, the PDE of the arithmetic Asian options in three-dimensions could be transformed into a two-dimension ordinary differential equation, and we have provided its analytical solution. The analytical solution for all types of the arithmetic Asian options can be obtained by changing the payoff function according to the type of the option that we need to price.

\section{REFERENCES}

1. Barucci E, Polidoro S, Vespri V (2001) Some results on partial differential equations and Asian options. Math Model Meth Appl Sci 11, 475-97.

2. Geman H, Yor M (1993) Bessel process, Asian options and perpetuities. Math Finance 3, 349-75.

3. Rogers L, Shi Z (1995) The value of an Asian option. J Appl Probab 32, 1077-88.

4. Zhang JE (2003) Theory of continuously-sampled Asian option pricing. J Futures Market 23, 535-60.

5. Vecer J (2001) A new PDE approach for pricing arithmetic average Asian option. J Comput Finance 4, 105-13.

6. Dubois F, Lelievre T (2004) Efficient pricing of Asian options by the PDE approach. J Comput Finance 8, 55-64.

7. Chen K, Lyuu Y (2007) Accurate pricing formula for Asian options. Appl Math Comput 188, 1711-24.

8. Dewynne JN, Shaw WT (2008) Differential equations and asymptotic solutions for arithmetic Asian options:
'Black-Scholes formulae' for Asian rate calls. J Appl Math 19, 353-91.

9. Yang Z, Oliver C-O, Menkens O (2011) Pricing and hedging of Asian options: quasi-explicit solutions via Malliavin calculus. Math Meth Oper Res 74, 93-120.

10. Cruz-Baez DI, Gonzalez-Rodrigues JM (2008) A different approach for pricing Asian options. Appl Math Lett 21, 303-6.

11. Erdelyi A, Mafnus W, Oberhettinger F, Tricomi FG (1954) Tables of Integral Transforms, Vol 1, McGraw Hill, New York. 